\title{
Increased periodontal inflammation in women with preterm premature rupture of membranes
}

\author{
Pascale F. M. Stadelmann • Sigrun Eick • Giovanni E. Salvi • Daniel Surbek • \\ Stefan Mohr • Walter Bürgin • Christoph A. Ramseier • Anton Sculean
}

Received: 21 July 2014 / Accepted: 14 November 2014 /Published online: 26 November 2014

(C) Springer-Verlag Berlin Heidelberg 2014

\begin{abstract}
Objectives To evaluate possible differences in periodontal inflammatory, microbiological and clinical parameters between women with preterm premature rupture of membranes (PPROM) and controls with uncomplicated pregnancies.

Materials and methods Fifty-six women (32 test (PPROM) and 24 controls (uncomplicated pregnancies)) were examined at three time-points (T1: gestational weeks 2035, T2: within $48 \mathrm{~h}$ after parturition, T3: 4-6 weeks after parturition). The examinations included assessment of the Periodontal Screening Index, collection of gingival crevicular fluid (GCF) and subgingival as well as vaginal bacterial sampling.

Results Periodontal inflammation was found to be higher in the test compared with the control group $(p<0.05)$ and decreased over time in both groups $(p<0.05)$. Microbiological outcomes showed no intergroup differences $(p>0.05)$ in prevalence of bacteria, but a decrease in subgingival periodontopathogens from $\mathrm{T} 1$ to $\mathrm{T} 2$ in the test group $(p<0.05)$ was observed. Interleukin (IL)-1 $\beta$ levels in GCF at $\mathrm{T} 2$ were not different between groups $(p>0.05)$. In women with PPROM, GCF levels of IL-8 $(p<0.05)$ and C-reactive protein $(p<0.05)$ were lower and IL-10 levels higher $(p<0.05)$ compared with controls.

Conclusions Periodontal inflammation is elevated during pregnancy and seems to be more pronounced in women with PPROM.
\end{abstract}

\footnotetext{
P. F. M. Stadelmann · S. Eick · G. E. Salvi $\cdot$ W. Bürgin •

C. A. Ramseier $\cdot$ A. Sculean $(\square)$

Department of Periodontology, School of Dental Medicine,

University of Bern, Freiburgstrasse 7, 3010 Bern, Switzerland

e-mail: anton.sculean@zmk.unibe.ch

D. Surbek $\cdot$ S. Mohr

Department of Obstetrics and Gynecology, University Hospital Bern and University of Bern, Bern, Switzerland
}

Clinical relevance The findings of the present study revealed an association between periodontal inflammation and PPROM, thus emphasizing the importance of optimizing self-performed oral hygiene in pregnant women.

Keywords PPROM · Periodontal inflammation · Periodontopathogenic bacteria - Gingival crevicular fluid . Inflammatory mediators $\cdot$ Adverse pregnancy outcomes

\section{Introduction}

Adverse pregnancy outcomes (APO), in particular preterm birth (PTB), continue to be a significant public health issue in both developed and developing countries. They represent approximately $10 \%$ of all live births worldwide [1], $11.54 \%$ in the USA [2] and $7.3 \%$ in Switzerland [3] and account for $28-75 \%$ of all perinatal deaths and over $50 \%$ of all severe developmental disability in children worldwide [4-7]. Therefore, the prevention of APO is an important goal for public health services warranting further research in its pathogenesis because, in a considerable number of cases of APO, the reasons for the disorder remain unclear. Forty to forty-five percent of PTB are caused by preterm labor and $25-30 \%$ by preterm premature rupture of membranes (PPROM), and 30 $35 \%$ are medically indicated [8]. PPROM represents a specific group of APO and is defined as premature rupture of membranes occurring prior to 37 weeks of gestation. PPROM occurs in approximately $3 \%$ of pregnancies and is responsible for a third of all PTBs [9].

Preclinical evidence in the golden hamster model indicated that challenge with Porphyromonas gingivalis into a subcutaneous chamber as a simulation of periodontal infection may lead to decreased fetal weight and increased embryolethality [10]. From a clinical point of view, periodontal disease has been linked to APO, including primarily PTB, low birth 
weight (LBW), PPROM, miscarriage, or pre-eclampsia. Current systematic reviews published on the topic of an association between APO and periodontal disease concluded that, despite a consistent association between periodontal disease and PTB or LBW, the findings should be interpreted with great caution because of heterogeneity among the studies [11, 12]. Variability among study designs, diagnostic methods and definitions of periodontal disease or APO makes it difficult to compare the published data. In addition, according to a recent review, there are no reductions in PTB after periodontal therapy in pregnant women in clinical trials [13]. In agreement, in a recent study, it was shown that periodontal therapy in pregnant women improved periodontal health without reducing the rate of preterm low birth weight [14]. However, the exact pathophysiological mechanism behind a possible association between APO and periodontal disease remains to be elucidated.

Several investigations reported a positive association between increased subgingival periodontopathogenic bacterial loads and APO [15-17], whereas other studies did not confirm these findings [18-20]. Furthermore, increased levels of inflammatory mediators (prostaglandin $(\mathrm{PG}) \mathrm{E}_{2}$, interleukin (IL)- $1 \beta$, tumor necrosis factor (TNF)- $\alpha$ ) in gingival crevicular fluid (GCF) in subjects with APO have been reported by several authors [15, 21-23]. In a recent systematic review, we concluded that a positive association between GCF inflammatory mediator levels and APO might exist, although these results have to be interpreted with caution because of the heterogeneity and variability among the studies and small sample sizes [24].

To the best of our knowledge, there are no studies investigating the association between periodontitis and PPROM in particular. Existing data always evaluated the disorder PPROM in combination with other causes for PTB like preterm labor [25-27]. Above all, there is a lack of knowledge about the prevalence of periodontopathogenic bacteria in subgingival biofilms and the levels of inflammatory mediators in GCF in women with PPROM. Therefore, the aim of the present study was to investigate the potential association between periodontal inflammation and PPROM.

\section{Materials and methods}

Study design and subject selection

This prospective case-control study was designed and conducted as collaboration between the Department of Periodontology (Dental school, University of Bern) and the Department of Obstetrics and Gynecology (University Hospital Bern and University of Bern). The study protocol was submitted to and approved by the Ethical Committee of the Canton Bern, Switzerland (KEK approval Nr. 091/10).
Participants were recruited at the Department of Obstetrics and Gynecology from November 2011 to August 2013, cases when presenting with PPROM and controls during regular gestational examinations. They were included after being informed about the study design and signing a written informed consent. Cases were defined as pregnant women with PPROM between the 20th and 35th gestational week. Controls were defined as women with uncomplicated pregnancies delivering at term ( $\geq 37$ gestational weeks).

The study design included three time-points of examination (T1, T2 and T3). These examinations included the assessment of the Periodontal Screening Index (PSI), collection of GCF and subgingival as well as vaginal bacterial sampling. T1 was set immediately after inclusion of the subjects (gestational weeks 20-35), T2 within $48 \mathrm{~h}$ after parturition and T3 4 6 weeks after parturition. Subjects missing two out of the three examinations were defined as dropouts.

Women with PPROM were managed as follows, according to the information and the standard protocol of the Department of Obstetrics and Gynecology: They routinely received antibiotic treatment after the diagnosis of PPROM (clindamycin or amoxicillin/clavulanic acid), which correlates with $\mathrm{T} 1$ in this group. Antibiotics were administered for 9 days (clindamycin $600 \mathrm{mg}$ intravenously (IV) every $8 \mathrm{~h}$ for $48 \mathrm{~h}$, then $300 \mathrm{mg}$ per orem (PO, or by mouth) every $8 \mathrm{~h}$ for 7 days or amoxicillin/clavulanic acid $1.2 \mathrm{~g}$ IV every $8 \mathrm{~h}$ for $48 \mathrm{~h}$, then $1 \mathrm{~g}$ PO every $12 \mathrm{~h}$ for 7 days). Tocolysis was administered for $48 \mathrm{~h}$ up to a maximum of 7 days. Antenatal glucocorticoids (intramuscular betamethasone $2 \times 12 \mathrm{mg}$ within $24 \mathrm{~h}$ ) were given to promote fetal pulmonary maturation. Pregnancy was ended by induction of labour or Cesarian section (according to clinical indication) at completion of 34 gestational weeks or before, for one of the following reasons: persistent labour, persistent vaginal bleeding, amnion infection syndrome or fetal distress (either on cardiotocography or on fetal Doppler examination) and placental abruption.

\section{Clinical periodontal examination}

Most of the examinations had to be conducted at the bedside at the Department of Obstetrics and Gynecology. Moreover, a majority of the subjects refused a detailed examination including full periodontal charts and oral radiographs at the Department of Periodontology. Thus, only the PSI could be recorded.

The PSI included assessment of periodontal probing at the mesiobuccal and distobuccal site of each tooth. Each sextant was assigned a score from 0 to $4(0, \mathrm{PD} \leq 3 \mathrm{~mm}$ and bleeding on probing (BOP)-negative; $1, \mathrm{PD} \leq 3 \mathrm{~mm}$ and $\mathrm{BOP}$-positive; $2, \mathrm{PD} \leq 3 \mathrm{~mm}$ and supra- or subgingival calculus or other plaque-retentive factors; 3 , PD $4-5 \mathrm{~mm} ; 4, \mathrm{PD} \geq 6 \mathrm{~mm}$ ). Mean PSI values and the percentage of sextants with a score 0 were calculated for each subject at all three time-points. 
Microbiological sampling

Subgingival plaque was collected from the mesiobuccal site of each first molar in all quadrants. If the first molar was missing, the second molar was used, and if the second molar was absent too, the second premolar was chosen. A sterile endodontic paper point (ISO 055, Dentsply Maillefer, Montigny Le Bretonneux, France) was inserted into the gingival crevice at each site until resistance was felt for $15 \mathrm{~s}$. Subsequently, the paper points were placed as a pooled sample into a screw-top plastic vial. In addition, a vaginal swab was provided by the Department of Obstetrics and Gynecology. Both samples were stored at $-20{ }^{\circ} \mathrm{C}$ until assayed for presence of periodontopathogens.

\section{GCF sampling}

GCF samples were collected from the same sites as subgingival plaque was sampled before. The collection was performed by means of sterile paper strips (Periopaper, Oraflow Inc, Smithtown, NY, USA), which were placed at the entrance of the crevice and left in place for $15 \mathrm{~s}$. Subsequently, the paper strips were transferred into a screw-top plastic vial and stored at $-80{ }^{\circ} \mathrm{C}$ until assayed.

Microbiological analysis

DNA was extracted by using Chelex method [28]. For detection of periodontopathogens, the microIDent ${ }^{\circledR}$ plus 11 test (Hain Lifescience, Nehren, Germany) was used according to the manufacturer's description. The test is able to identify 11 periodontopathogenic bacterial species after two polymerase chain reaction runs and a subsequent reverse hybridization.

Analysis was made qualitatively for each species. Subsequently, the samples that were positive for Aggregatibacter actinomycetemcomitans, $P$. gingivalis, Tannerella forsythia and/or Treponema denticola were counted. Moreover, in an additional analysis, these four species were added to the other seven analyzed microorganisms or complexes (Prevotella intermedia, Parvimonas micra, Fusobacterium nucleatum / necrophorum, Campylobacter rectus, Eubacterium nodatum, Eikenella corrodens and Capnocytophaga species).

Furthermore, subgingival and vaginal bacterial samples being tested positive for A. actinomycetemcomitans were analyzed for presence of genotypes b and c, as described recently [29].

Analysis of inflammatory mediators (GCF samples)

Before analyzing, GCF samples were eluted at $4{ }^{\circ} \mathrm{C}$ overnight into $750 \mu \mathrm{l}$ phosphate-buffered saline containing proteinase inhibitors (Sigma-Aldrich, St. Louis, MO, USA). From the eluates, the levels of IL- $1 \beta$, IL-6, IL-8, IL-10 and C-reactive protein (CRP) were determined by using commercially available enzyme-linked immunosorbent assay kits ( R \& D Systems Europe Ltd., Abingdon, UK) according to the manufacturer's instructions. The detection levels of the kits were $2 \mathrm{pg} / \mathrm{site}$ (CRP, $10 \mathrm{pg} / \mathrm{site})$.

\section{Null hypothesis}

No statistically significant differences are observed with respect to GCF inflammatory mediators, prevalence of subgingival and vaginal periodontopathogens and clinical periodontal parameters between the test (PPROM) and control groups at any time-point.

Data analysis

The primary outcome variable was IL- $1 \beta$ in GCF at T2. Secondary outcome variables included the levels of IL-1 $\beta$ at $\mathrm{T} 1$ and $\mathrm{T} 3$ as well as the remaining inflammatory mediators analyzed (IL-6, IL-8, IL-10 and CRP), PSI and the prevalence of subgingival and vaginal periodontopathogenic bacteria at any time-point.

Sample-size calculation was based on the comparison of GCF IL-1 $\beta$ levels between mild preeclampsia and controls within $48 \mathrm{~h}$ preceding delivery reported by Canakci et al. [30]. Following their results, a total sample size of 22 women using $\alpha$ of 0.05 would result in a power of 0.95 . However, because of the expected high rate of dropouts and the conditions not having been described to date in PPROM, a sample size of 35 women per group was scheduled for T1.

Statistical analysis was performed by using SAS ${ }^{\circledR} 9.4$ (SAS Institute Inc. Cary, NC, USA) and SPSS 21.0 (SPSS Statistics, IBM Corporation, New York, USA).

Qualitative data were analyzed by Fisher's exact test for independent groups and McNemar test for comparing timepoints within groups (prevalence of bacteria). All other data were analyzed by non-parametric tests, Mann-Whitney $U$ test for independent groups and Wilcoxon matched pairs signed rank test for comparing time-points within groups. The level of significance was set at a $p=0.05$.

\section{Results}

Subject selection

In total, 70 subjects ( 35 cases, 35 controls) were recruited, from which 14 dropped out for various reasons (Fig. 1). In the test group, five subjects dropped out because of either stillbirth (1), delivery in another hospital (1) or not showing up for two examinations (3). The reasons for the nine dropouts in the control group were preeclampsia (1), stillbirth (1), delivery in another hospital (2) or not showing up for two examinations 
Fig. 1 Subject recruitment: flow chart indicating initial subject number, numbers of dropouts and final subject number

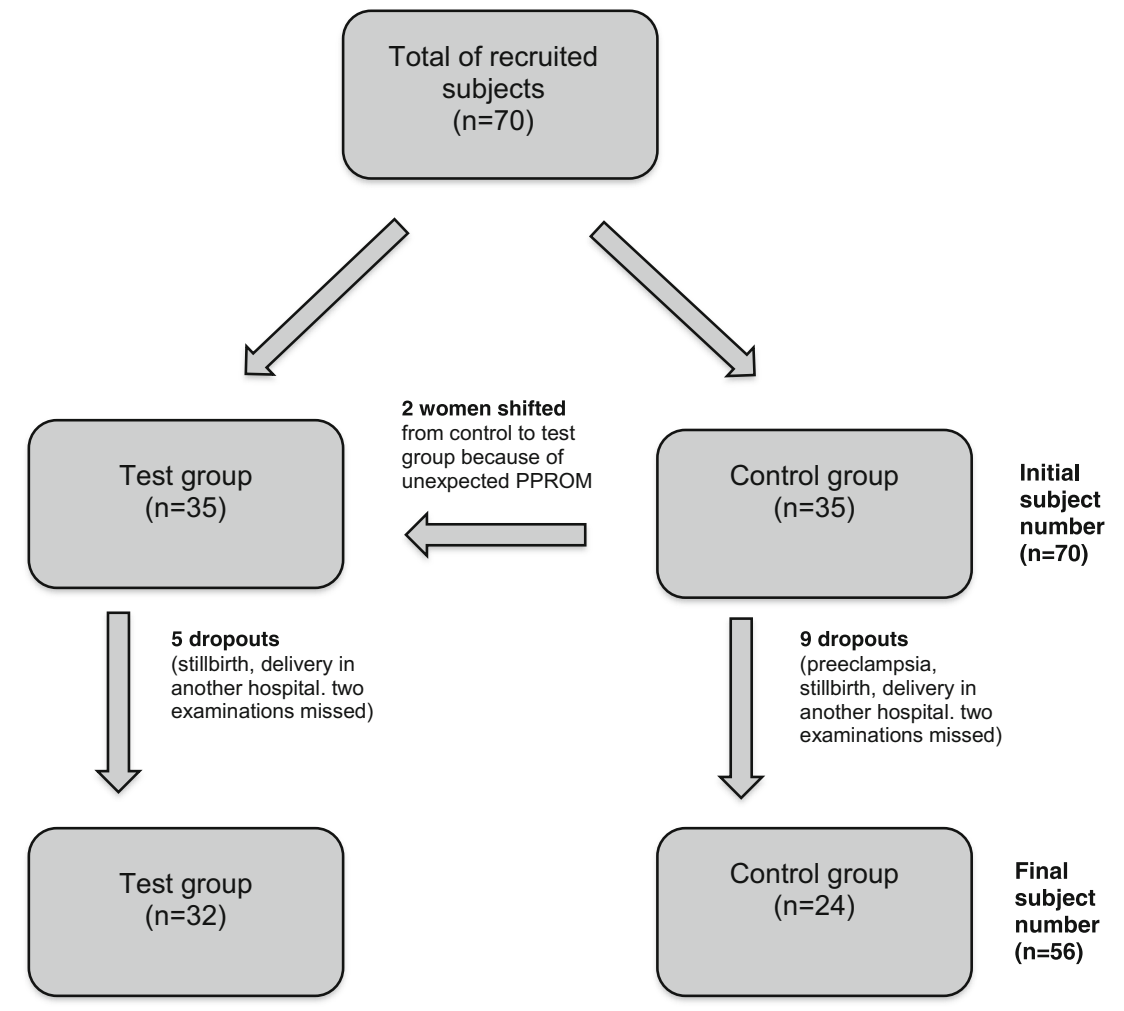

(5). Furthermore, two women originally assigned to the control group were shifted to the test group because of an unexpected incidence of PPROM.

Finally, 56 subjects were included in the analysis, 32 in the test and 24 in the control group. All subjects underwent the examinations at T1. At T2 and T3, 31 and 25 subjects of the test group and 23 and 22 subjects of the control group were available, respectively.

As mentioned above, a majority of the women refused a detailed examination at the Department of Periodontology. Thus, no full periodontal charts or oral radiographs could be obtained due to limited oral examination facilities at the Department of Obstetrics and Gynecology.

\section{Baseline characteristics of subjects}

Baseline characteristics are presented in Table 1. Mean age $(p=0.5814)$ and the percentage of non-Caucasians ( $p=$ 0.3243 ) were not statistically significantly different between groups. Among the non-Caucasian subjects, there was 1 $(3.13 \%)$ versus $2(8.33 \%)$ African, 1 (3.13\%) versus 2 $(8.33 \%)$ Asian and 0 versus $2(8.33 \%)$ Hispanic in the test versus control group.

Neither systemic diseases (hypertension, obesity, hyperthyroidism, hypothyroidism, hepatitis B) $(p=0.2680)$, nor smoking $(p=1.0000)$, nor complications during previous pregnancies (miscarriage, preeclampsia, PPROM, intrauterine fetal death) ( $p=0.0730)$ nor primipara $(p=0.7880)$ were distributed statistically significantly differently in the two groups.

Obstetric outcomes

Obstetric outcomes are presented in Table 1. PPROM (test subjects) occurred at 30.93 (range, 23-35) gestational weeks. In median, subjects in the test and control groups delivered at 32.57 and 39.21 gestational weeks $(p<0.001)$, respectively, whereas birth weights were 1,870 and $3,418 \mathrm{~g}(p<0.001)$, respectively.

\section{Clinical periodontal outcomes}

Results of the clinical periodontal examination are presented in Fig. 2. Median values of the mean PSI at T1, T2 and T3 were 2.17, 1.50 and 1.17 in the test group and $1.17,1.00$ and 0.83 in the control group, respectively. They were statistically significantly higher in the test group compared with the control group at all three time-points (T1, $p=0.006$; T2, $p=0.037$; T3, $p=0.044$ ). In the test group, the mean PSI was statistically significantly different between all time-points $(p=0.0031$ for $\mathrm{T} 1-\mathrm{T} 2, p=0.0025$ for $\mathrm{T} 1-\mathrm{T} 3, p=0.0259$ for $\mathrm{T} 2-\mathrm{T} 3$ ) whereas, in the control group, there was a statistically significant difference between T1 and T3 $(p=0.0008)$ as well as T2 and T3 $(p=0.0116)$, but not between T1 and T2 $(p=0.2696)$. 
Table 1 Baseline characteristics and obstetric outcomes of subjects

\begin{tabular}{|c|c|c|c|}
\hline & Test subjects $(n=32)$ & Control subjects $(n=24)$ & \\
\hline \multicolumn{4}{|l|}{ Baseline characteristics } \\
\hline Mean age (years) & $32.66(20-41)$ & $33.55(22-40)$ & $p=0.5814$ \\
\hline \multicolumn{4}{|l|}{ Ethnic origin Caucasian } \\
\hline Caucasian & $30(93.75 \%)$ & $18(75.00 \%)$ & $p=0.3243$ \\
\hline Non-Caucasian & $2(6.25 \%)$ & $6(25.00 \%)$ & \\
\hline Systemic diseases & $3(9.38 \%)$ & $5(20.83 \%)$ & $p=0.2680$ \\
\hline Hypertension & 0 & 0 & \\
\hline Obesity & $2(6.25 \%)$ & $3(12.5 \%)$ & \\
\hline Hyperthyreosis & $1(3.13 \%)$ & 0 & \\
\hline Hypothyreosis & 0 & $2(8.33 \%)$ & \\
\hline Hepatitis B & $1(3.13 \%)$ & $1(4.17 \%)$ & \\
\hline Smoking & $2(6.25 \%)$ & $1(4.17 \%)$ & $p=1.0000$ \\
\hline Complications during previous pregnancies & $9(28.13 \%)$ & $9(37.50 \%)$ & $p=0.0730$ \\
\hline Primipara & $20(62.50 \%)$ & $14(58.33 \%)$ & $p=0.7880$ \\
\hline \multicolumn{4}{|l|}{ Obstetric outcomes } \\
\hline Delivery (gestational weeks) & $32.57(27-35)$ & $39.21(36-41)$ & $p<0.001$ \\
\hline Birth weight (g) & $1870(535-2870)$ & $3418(2555-4260)$ & $p<0.001$ \\
\hline
\end{tabular}

Median values and ranges for quantitative data, numbers and percentages for qualitative data and values of significance are indicated for test and control groups ( $p$ values $\geq 0.05=$ n.s.)

The median percentages of sextants with a PSI score 0 per patient at $\mathrm{T} 1, \mathrm{~T} 2$ and $\mathrm{T} 3$ were each $0 \%$ in the test group and $16.67 \%, 33.33 \%$ and $33.33 \%$ in the control group, respectively. This percentage was statistically significantly higher in the control group compared with the test group at all time-points (T1, $p=0.003$; T2, $p=0.003$; T3, $p=0.015$ ).
Fig. 2 Clinical periodontal outcomes: mean PSI (a) and the percentage of sextants with PSI score $0(\mathbf{b})$ are presented at the different time-points (T1, T2, T3) for test and control groups. Difference between test group and controls, ${ }^{\natural} p<0.05 ;{ }^{\natural \pi} p<0.01$; difference within groups, ${ }^{*} p<0.05 ; * * p<0.01$
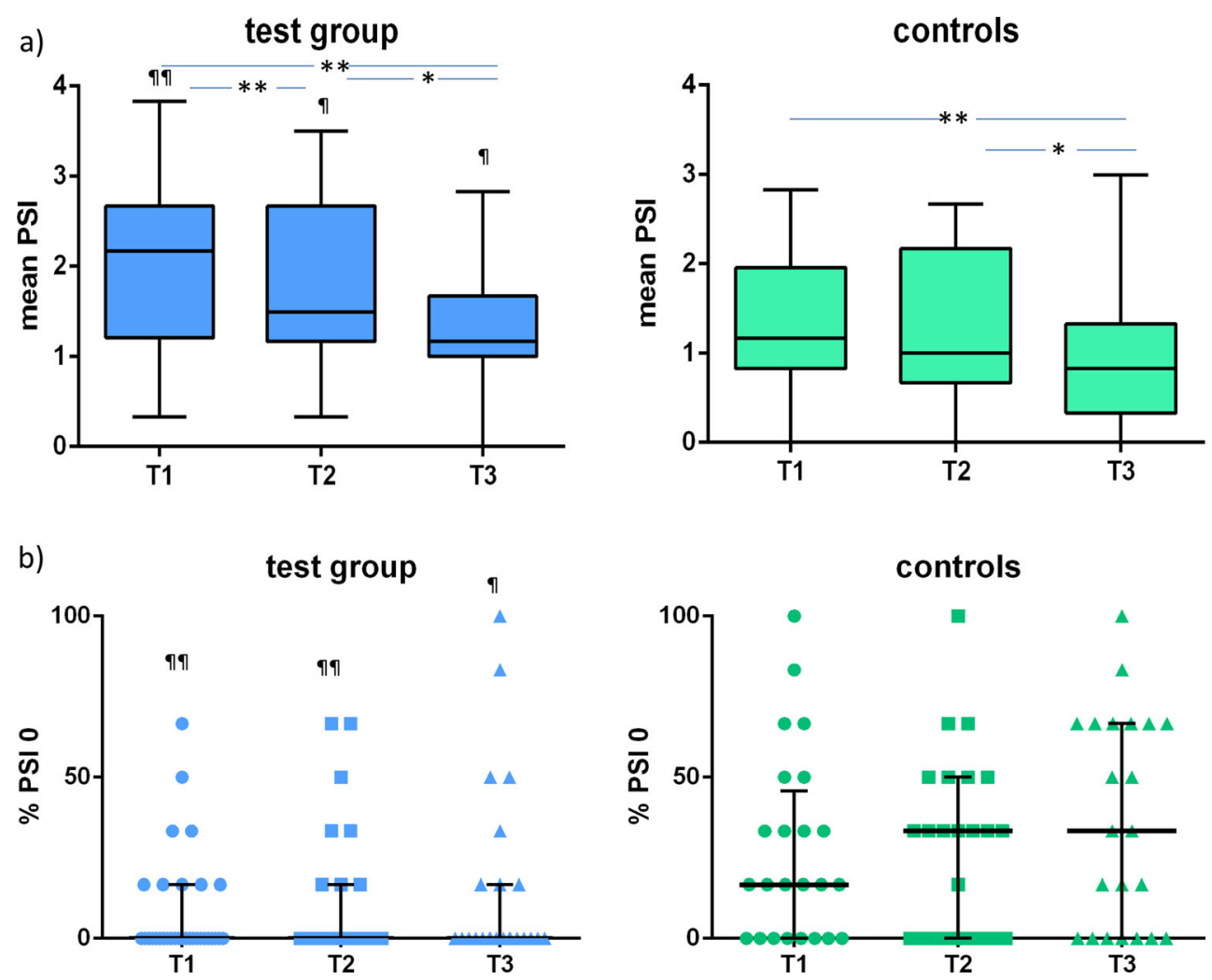
In 16 of $45(35.56 \%)$ women, a PSI score 3 or 4 at T1 decreased to a PSI score $\leq 2$ at T3.

\section{Microbiological outcomes}

The prevalence of periodontopathogenic bacteria in subgingival and vaginal samples is presented in Table 2. There were no statistically significant differences between groups at any timepoint $(p>0.05)$. Over time, the prevalence of grouped subgingival periodontopathogenic bacteria did not change in the control group $(p>0.05)$. In the test group, there was a statistically significant decrease from $\mathrm{T} 1$ to $\mathrm{T} 2$ for the microbiological group of major periodontopathogens (A. actinomycetemcomitans, $P$. gingivalis, T. denticola, T. forsythia $)(p=0.0313)$ and also for the group of all analyzed bacteria ( $p=0.0039)$. In the vaginal samples, there was a statistically significant increase in the test group from T1 to T3 for the group of all analyzed bacteria $(p=0.0391)$.

In six vaginal samples, $A$. actinomycetemcomitans was identified. Moreover, $P$. gingivalis was found in three and T. denticola and $T$. forsythia in two vaginal samples, respectively.

A. actinomycetemcomitans was detected both subgingivally and in vaginal fluid in four women. These samples were further subjected to genotype analysis. In subgingival samples, genotype b was found two times, genotype $\mathrm{c}$ once and neither genotype b or c once, whereas vaginal genotypes were neither $\mathrm{b}$ nor $\mathrm{c}$ in all cases.

\section{Outcomes of GCF inflammatory mediators}

Data reporting the levels of inflammatory mediators in GCF are presented in Fig. 3. IL-1 $\beta$ levels in GCF at T2 were not significantly different between groups but seemed to be higher in the control group $(p=0.053)$. At T1, statistically significantly lower levels of IL-8 $(p=0.008)$ and CRP $(p=0.008)$ and higher levels of IL-10 ( $p=0.004)$ were measured in the test compared with the control group. Also at T3, levels of IL-10 were statistically significantly higher $(p=0.029)$ in the test compared with the control group. A statistically significant decrease in IL-1 $\beta$ levels from T1 to T2 $(p<0.001)$ and an increase from T2 to T3 $(p=0.046)$ were found in the test group. However, the CRP levels were statistically significantly higher at T2 than at T1 $(p=0.002)$. In the control group, the CRP levels decreased statistically significantly from $\mathrm{T} 1$ to $\mathrm{T} 3$ $(p=0.015)$ and from T2 to T3 $(p=0.002)$.

Data about the intergroup comparison of the change of inflammatory mediators over time are presented in Table 3. Significant differences between groups were found for IL- 8 with a greater increase from T2 to T3 in the test group $(p=0.0092)$ and a difference between the increase in the test group and the decrease in the control group from T1 to T3 $(p=0.0283)$. Furthermore, IL$1 \beta$ showed a greater decrease from T1 to T2 $(p=0.0047)$, whereas a smaller one was found for CRP from T2 to T3 $(0.0251)$ as well as from T1 to T3 $(p=0.0116)$ in the test group.

Analysis of the ratios between IL-10 and IL-1 $\beta$ and between IL-10 and CRP showed in part significantly higher values in the test group (Table 4).

\section{Discussion}

The present investigation was designed as a prospective casecontrol study in pregnant women with examinations at three time-points, at gestational weeks 20-35, right after parturition and 4-6 weeks after parturition. Cases were defined as pregnant women with PPROM between the 20th and 35th gestational week, whereas controls exhibited uncomplicated pregnancies. Although various studies have investigated an association between periodontitis and APO in general so far, to the best of our knowledge, this study is the first to prove an association between periodontal inflammation and PPROM specifically.

Table 2 Microbiological outcomes

\begin{tabular}{|c|c|c|c|c|c|c|c|}
\hline & & \multicolumn{3}{|c|}{ Subgingival } & \multicolumn{3}{|c|}{ Vaginal } \\
\hline & & $n$ & All species & Major species & $n$ & All species & Major species \\
\hline \multirow[t]{3}{*}{ Test } & $\mathrm{T} 1$ & 32 & $28(87.50 \%)^{* *}$ & $13(40.63 \%)^{*}$ & 31 & $5(16.13 \%)^{*}$ & $4(12.90 \%)$ \\
\hline & $\mathrm{T} 2$ & 31 & $18(58.06 \%)^{* *}$ & $7(22.58 \%)^{*}$ & 30 & $8(26.67 \%)^{*}$ & $6(20.0 \%)$ \\
\hline & $\mathrm{T} 3$ & 24 & $20(83.33 \%)$ & $2(8.33 \%)$ & 25 & $9(36.00 \%)^{*}$ & $6(24.00 \%)$ \\
\hline \multirow[t]{3}{*}{ Control } & $\mathrm{T} 1$ & 24 & $19(79.17 \%)$ & $7(29.17 \%)$ & 23 & $1(4.35 \%)$ & $1(4.35 \%)$ \\
\hline & $\mathrm{T} 2$ & 23 & $15(65.22 \%)$ & $4(17.39 \%)$ & 23 & $3(13.04 \%)$ & $2(8.70 \%$ \\
\hline & $\mathrm{T} 3$ & 22 & $19(86.35 \%)$ & $6(27.27 \%)$ & 22 & $3(13.64 \%)$ & $1(4.55 \%)$ \\
\hline
\end{tabular}

Prevalence and percentage of all species (A. actinomycetemcomitans, P. gingivalis, T. forsythia, T. denticola, P. intermedia, P. micra, F. nucleatum/ necrophorum, C. rectus, E. nodatum, E. corrodens, Capnocytophaga species) and major species (A. actinomycetemcomitans, $P$. gingivalis, $T$. forsythia, T. denticola) of periodontopathogenic bacteria analyzed in subgingival and vaginal samples are presented at the different time-points (T1, T2, T3) for test and control groups; no significant differences between test and control groups; difference within groups

${ }^{*} p<0.05 ;{ }^{*} p<0.01$ 
a)

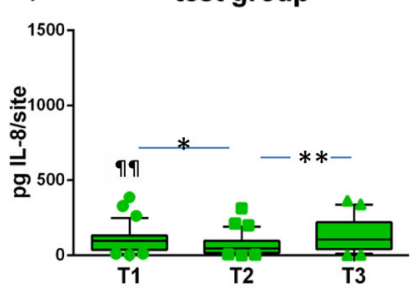

b) test group
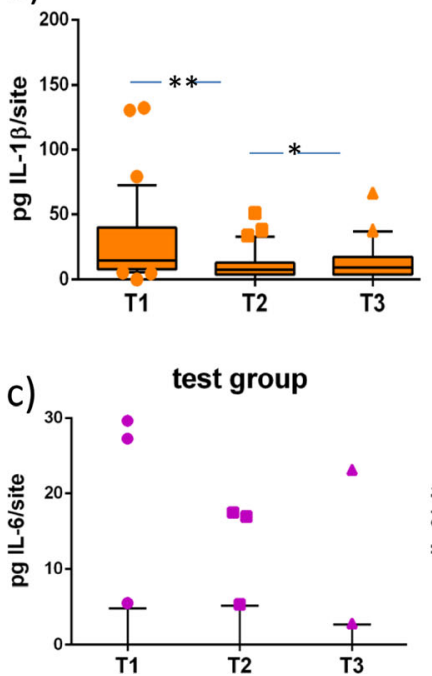

controls

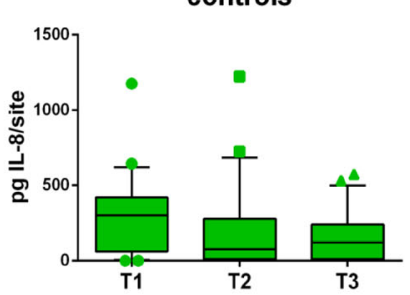

controls

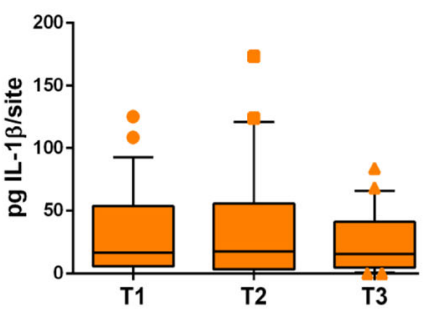

d)
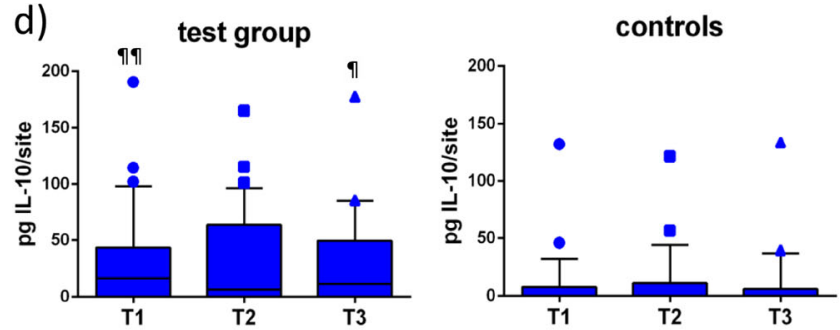

e)
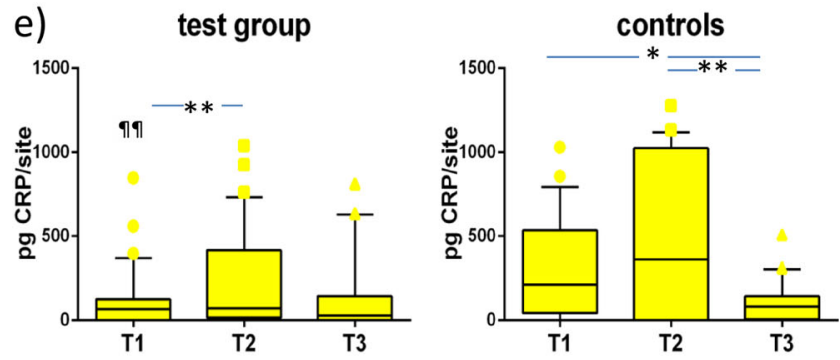

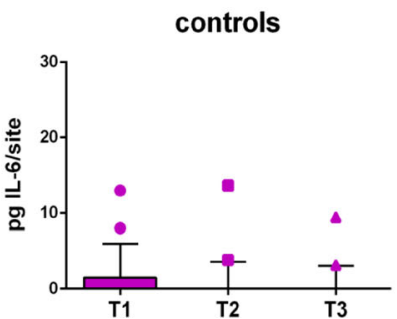

Fig. 3 GCF inflammatory mediators: levels for IL-8 (a), IL-1 $\beta$ (b), IL-6 (c), IL-10 (d) and CRP (e) are presented at the different time-points (T1, T2, T3) for test and control groups. Difference between test group and controls, ${ }^{\natural} p<0.05 ;{ }^{{ }^{\natural}} p<0.01$; difference within groups, ${ }^{*} p<0.05 ; * * p<0.01$

A drawback of the study design might be the differing protocol between test and control groups including the intake of antibiotics in the test group. Administration of antibiotics is part of the standard protocol in women diagnosed with PPROM. Hence, a potential bias in the test group cannot be excluded. However, the results in both groups show similar tendencies concerning the development over time which may underline and justify the main statements and conclusions of the study.

The fact that in $35.56 \%$ of the women a PSI score 3 or 4 at T1 decreased to a PSI score $\leq 2$ at T3 and no periodontal treatment was delivered leads to the assumption that the increased probing depths at $\mathrm{T} 1$ resulted mainly from the

Table 3 Intergroup comparison of the change of inflammatory mediators between different time-points (T1, T2, T3)

\begin{tabular}{llll}
\hline & T1-T2 & T2-T3 & T1-T3 \\
\hline IL-8 & n.s. & 0.0092 & 0.0283 \\
IL-1 $\beta$ & 0.0047 & n.s. & n.s. \\
IL-6 & n.s. & n.s. & n.s. \\
IL-10 & n.s. & n.s. & n.s. \\
CRP & n.s. & 0.0251 & 0.0116 \\
\hline
\end{tabular}

Values of significance are presented ( $p$ values $\geq 0.05=$ n.s., not significant) condition due to the current pregnancy gingivitis rather than from a true periodontitis. Considering these circumstances, a separate analysis was conducted with the aim of obtaining more differentiated information by distinguishing the proportions of inflamed versus non-inflamed tissues in each subject. Therefore, the percentage of sextants with a PSI score 0 per patient was additionally evaluated.

A further limitation of the present study was the fact that no full periodontal charts could be obtained. In pregnant women with a high degree of gingival inflammation and presumable pseudopockets, full periodontal charts allow a precise distinction between gingivitis and periodontitis. Although previous studies in this field were also using partial mouth recordings, it has to be considered that this method of assessing periodontal parameters only provides limited information about extent and severity of periodontal disease, e.g., the score is only representative for the maximum probing depth in the sextant, and the numbers of sites with pathological probing depths as well as the presence of pseudopockets are not considered.

Nevertheless, the finding of more pronounced periodontal inflammation in the test group expressed by higher mean PSI values and a smaller percentage of sextants with a PSI score 0 corroborates the hypothesis of an association between periodontal status and APO [11]. Despite the relatively small sample size, these results suggest an association between 
Table 4 Ratios of IL-10/IL-1 $\beta$ and IL-10/CRP

\begin{tabular}{lllllllllll}
\hline & \multicolumn{2}{c}{$\mathrm{T} 1$} & \multicolumn{2}{c}{$\mathrm{T} 2$} & & $\mathrm{~T} 3$ & $\mathrm{~T} 1-\mathrm{T} 2$ & $\mathrm{~T} 2-\mathrm{T} 3$ & $\mathrm{~T} 1-\mathrm{T} 3$ \\
\hline IL-10/IL-1 $\beta$ & Test & $0.99(0.01-11.25)$ & & $1.00(0.05-24.09)$ & & $2.77(0.10-19.41)$ & & n.s. & n.s. & n.s. \\
& Control & $0.14(0.02-8.82)$ & n.s. & $0.17(0.01-14.08)$ & 0.034 & $0.21(0.03-4.62)$ & 0.006 & n.s. & n.s. & n.s. \\
IL-10/CRP & Test & $0.66(0.00-15.94)$ & & $0.12(0.00-17.23)$ & & $0.75(0.01-18.18)$ & 0.001 & 0.022 & n.s. \\
& Control & $0.01(0.00-9.43)$ & 0.002 & $0.01(0.00-12.38)$ & n.s. & $0.04(0.00-9.68)$ & 0.018 & n.s. & n.s. & n.s. \\
\hline
\end{tabular}

Median values and ranges are presented at the different time-points (T1, T2, T3) as well as intergroup and intragroup comparisons. Values of significance are indicated ( $p$ values $\geq 0.05=$ n.s., not significant)

periodontal inflammation and PPROM in particular, which has never been investigated so far. It must be emphasized, however, that there were no generalized severe periodontitis cases in the present study population (no subject of the test and only 1 subject $(4.17 \%)$ of the control group showed at least one PSI score 4 at T3).

Longitudinally, the mean PSI score decreased, whereas the percentage of sextants with a PSI score 0 increased from T1 to T3. An increase in the severity and prevalence of gingival inflammation during pregnancy has been reported by several authors [31, 32] and reviewed by Figuero et al. [33], being associated with a concomitant increase in probing depth [34, 35]. Gingivitis seems to resolve without any active therapy postpartum [36, 37]. The mechanism of increased inflammation during pregnancy is suggested to be linked with hormonal effects on the vascular system [38], the immune response [39], supra- and subgingival biofilms $[32,36,40]$ and cells of the periodontium [41].

The microbiological outcomes are in agreement with the clinical findings of more pronounced periodontal inflammation during pregnancy in general and also in the test group. The tendency for a higher prevalence of subgingival periodontopathogenic bacteria at $\mathrm{T} 1$ and $\mathrm{T} 2$ is in correlation with the higher PSI scores in the test group, and it also corroborates the hypothesis of an association between periodontal inflammation and PPROM. Several previous studies reported an association between higher loads of subgingival periodontopathogenic bacteria and risk for PTB [15-17], whereas others could not confirm this finding [19, 20].

A tendency for a decrease in the prevalence of major periodontopathogens in subgingival plaque was found in both groups from $\mathrm{T} 1$ to $\mathrm{T} 3$, as well as a statistically significant reduction from $\mathrm{T} 1$ to $\mathrm{T} 2$ in the test group. The gynecological protocol in women with PPROM included an intake of antibiotics, which might also suppress bacterial growth within the periodontium. There are different reports on the changes in prevalence or proportions of periodontopathogenic bacteria in subgingival biofilms throughout pregnancy and after delivery, failing to report any changes [36] or describing decreasing proportions of $A$. actinomycetemcomitans, $P$. gingivalis and T. forsythia after delivery [40].
The composition of the subgingival microbiota during pregnancy might be influenced by the increased levels of hormones. $P$. intermedia is able to take up and metabolize estradiol and progesterone [42], and consequently, higher loads of this species are found during pregnancy [43]. C. rectus was present in higher loads in the saliva of pregnant women compared with non-pregnant women, correlating with higher estradiol levels [44]. In the present investigation, P. intermedia and $C$. rectus were detected in $5(8.9 \%)$ and $17(30.4 \%)$ of 56 women at $\mathrm{T} 1$ and in $0(0 \%)$ and $6(16.2 \%)$ of 37 women at T3.

Bacteria known to be associated with periodontitis were also detected in vaginal samples. In four women, subgingival as well as vaginal samples were tested positively for A. actinomycetemcomitans. However, genotyping excluded in three of the four cases subgingivally and vaginally matching strains, which underlines a similarity but not an identity of vaginal and subgingival microbiota. Interestingly, in the context of similarities between the microbiota in different body site niches, a current investigation concluded that the placental microbiome profiles were most akin to the human oral microbiome, and no similarity between the placental and vaginal microbiomes was evident [45].

Several studies have reported an association between different elevated GCF inflammatory mediator levels (IL- $1 \beta$, IL6 , TNF- $\alpha, \mathrm{PGE}_{2}$ ) and APO [15, 21, 22, 30, 46, 47], but, to the best of our knowledge, there are no existing studies investigating GCF inflammatory mediator levels in a specific sample of women with PPROM. In the present study, GCF levels of pro-inflammatory mediators (IL-8, CRP and in tendency IL$1 \beta)$ were lower in women with PPROM than in controls. This result seems to be in contrast with several other studies, where significantly higher GCF levels of IL- $1 \beta$, for instance, were found in women with APO compared with controls [15, 21, $22,30,46]$. Only the outcomes of a study by Noack et al. [19] did not confirm this. In addition to the pro-inflammatory mediators, the GCF level of IL-10 was measured, which, according to the current literature, has not been analyzed yet in association with APO or PPROM in particular. IL-10 is one of the most important anti-inflammatory cytokines and is mainly produced by monocytes and Th2-lymphocytes and during pregnancy also by gestational tissues. It decreases the 
production of pro-inflammatory cytokines such as IL- $1 \beta$, IL-8 and TNF- $\alpha[48,49]$. IL-10 obviously plays an important role in the physiological birth process as well as in preterm delivery. Gotsch et al. [50] found significantly higher amniotic fluid IL-10 concentrations in women in labour compared with women not in labour at term and also in women delivering preterm versus term. In addition, intra-amniotic infection (preterm and term) was associated with increased concentrations of amniotic fluid IL-10. Recent investigations also reported on IL-10 as a good predictor for amnionitis and funisitis in women with PPROM, confirming elevated IL-10 levels in PPROM associated with intra-amniotic infection [51, 52]. Furthermore, serum IL-10 levels in combination with RANTES and cervical length were suggested to be most predictive for spontaneous delivery in women with preterm labour [53]. The mentioned findings suggest that IL-10 plays a role in the mechanisms of labour (term and preterm) as well as in the host response to intrauterine infection, and according to the present outcomes, elevated IL-10 levels can be found also in GCF in women with a risk for APO, specifically PPROM.

In addition, a significant decrease in IL-1 $\beta$ levels from T1 to T2 was found in the test group, which is related to the observed general decrease in clinical periodontal inflammation and the hypothesis of more pronounced inflammation during pregnancy. This change was furthermore found to be significantly greater compared with the control group. Importantly, this decrease has to be considered and interpreted in the context of the antibiotic treatment in the test group, which might influence the inflammatory response in the periodontium.

\section{Conclusions}

Periodontal inflammation is generally elevated during pregnancy and more pronounced in women with PPROM. The elevated GCF levels of IL-10 in women with PPROM reflect a potential role of this cytokine in anti-inflammatory response with respect to the process of PTB. Further investigations in larger study cohorts are needed to confirm a potential association between periodontal inflammation and PPROM. The clinical relevance from the present findings underlines the importance of optimizing selfperformed oral hygiene in pregnant women, as they revealed, for the first time, an association between periodontal inflammation and PPROM.

Acknowledgements The authors wish to acknowledge Professor Rutger Persson for his useful advices during initiation of this study and to express their appreciation for the laboratory work of Stéphanie Larti, Anna Magdon and Marianne Weibel. They are also grateful to the Department of Obstetrics and Gynecology, University Hospital Bern, the midwives and doctors and the women having participated in this study.
Source of funding and conflict of interest This study has been funded by a grant from the Walter Haefner Stiftung, by the Clinical research fund of the Department of Obstetrics and Gynecology, University Hospital Bern and University of Bern, and by the Department of Periodontology, School of Dental Medicine, University of Bern. The authors report no conflicts of interest related to this study.

\section{References}

1. Beck S, Wojdyla D, Say L, Betran AP, Merialdi M, Requejo JH, Rubens C, Menon R, Van Look PF (2010) The worldwide incidence of preterm birth: a systematic review of maternal mortality and morbidity. Bull World Health Organ 88(1):31-38

2. Hamilton BE, Martin JA, Ventura SJ (2013) Births: preliminary data for 2012. Natl Vital Stat Rep 62(3):1-20

3. Bundesamt für Statistik (2012) Gesundheit der Neugeborenen. http:// www.bfs.admin.ch/bfs/portal/de/index/themen/14/02/03/key/04. $\mathrm{html}$

4. Smith GC, Fretts RC (2007) Stillbirth. Lancet 370(9600):1715-1725

5. Lawn JE, Cousens S, Zupan J (2005) 4 million neonatal deaths: when? Where? Why. Lancet 365(9462):891-900

6. Callaghan WM, MacDorman MF, Rasmussen SA, Qin C, Lackritz EM (2006) The contribution of preterm birth to infant mortality rates in the United States. Pediatrics 118(4):1566-1573

7. Goldenberg RL, Culhane JF, Iams JD, Romero R (2008) Epidemiology and causes of preterm birth. Lancet 371(9606):75-84

8. Tucker JM, Goldenberg RL, Davis RO, Copper RL, Winkler CL, Hauth JC (1991) Etiologies of preterm birth in an indigent population: is prevention a logical expectation. Obstet Gynecol 77(3):343-347

9. Mercer BM (2003) Preterm premature rupture of the membranes. Obstet Gynecol 101(1):178-193

10. Collins JG, Windley HW 3rd, Arnold RR, Offenbacher S (1994) Effects of a Porphyromonas gingivalis infection on inflammatory mediator response and pregnancy outcome in hamsters. Infect Immun 62(10):4356-4361

11. Chambrone L, Guglielmetti MR, Pannuti CM, Chambrone LA (2011) Evidence grade associating periodontitis to preterm birth and/or low birth weight: I. A systematic review of prospective cohort studies. J Clin Periodontol 38(9):795-808

12. Ide M, Papapanou PN (2013) Epidemiology of association between maternal periodontal disease and adverse pregnancy outcomessystematic review. J Periodontol 84(4 Suppl):S181-S194

13. Chambrone L, Pannuti CM, Guglielmetti MR, Chambrone LA (2011) Evidence grade associating periodontitis with preterm birth and/or low birth weight: II: a systematic review of randomized trials evaluating the effects of periodontal treatment. J Clin Periodontol 38(10):902-914

14. Weidlich P, Moreira CH, Fiorini T, Musskopf ML, da Rocha JM, Oppermann ML, Aass AM, Gjermo P, Susin C, Rosing CK, Oppermann RV (2013) Effect of nonsurgical periodontal therapy and strict plaque control on preterm/low birth weight: a randomized controlled clinical trial. Clin Oral Investig 17(1):37-44

15. Offenbacher S, Jared HL, O'Reilly PG, Wells SR, Salvi GE, Lawrence HP, Socransky SS, Beck JD (1998) Potential pathogenic mechanisms of periodontitis associated pregnancy complications. Ann Periodontol 3(1):233-250

16. Mitchell-Lewis D, Engebretson SP, Chen J, Lamster IB, Papapanou PN (2001) Periodontal infections and pre-term birth: early findings from a cohort of young minority women in New York. Eur J Oral Sci 109(1):34-39 
17. Lin D, Moss K, Beck JD, Hefti A, Offenbacher S (2007) Persistently high levels of periodontal pathogens associated with preterm pregnancy outcome. J Periodontol 78(5):833-841

18. Madianos PN, Lieff S, Murtha AP, Boggess KA, Auten RL Jr, Beck JD, Offenbacher S (2001) Maternal periodontitis and prematurity. Part II: maternal infection and fetal exposure. Ann Periodontol 6(1): $175-182$

19. Noack B, Klingenberg J, Weigelt J, Hoffmann T (2005) Periodontal status and preterm low birth weight: a case control study. J Periodontal Res 40(4):339-345

20. Africa CWJ, Kayitenkore J, Bayingana C (2010) Examination of maternal gingival crevicular fluid for the presence of selected periodontopathogens implicated in the pre-term delivery of low birth weight infants. Virulence 1(4):254-259

21. Konopka T, Rutkowska M, Hirnle L, Kopec W, Karolewska E (2003) The secretion of prostaglandin E2 and interleukin 1-beta in women with periodontal diseases and preterm low-birth-weight. Bull Group Int Rech Sci Stomatol Odontol 45(1):18-28

22. Carta G, Persia G, Falciglia K, Iovenitti P (2004) Periodontal disease and poor obstetrical outcome. Clin Exp Obstet Gynecol 31(1):47-49

23. Tarannum F, Faizuddin M, Madaiah H (2011) Gingival crevicular fluid prostaglandin E2 level as a predictor of preterm low birth weight: a pilot investigation. J Oral Sci 53(3):293-300

24. Stadelmann P, Alessandri R, Eick S, Salvi GE, Surbek D, Sculean A (2013) The potential association between gingival crevicular fluid inflammatory mediators and adverse pregnancy outcomes: a systematic review. Clin Oral Investig 17(6):1453-1463

25. Abati S, Villa A, Cetin I, Dessole S, Luglie PF, Strohmenger L, Ottolenghi L, Campus GG (2013) Lack of association between maternal periodontal status and adverse pregnancy outcomes: a multicentric epidemiologic study. J Matern Fetal Neonatal 26(4): 369-372

26. Nabet C, Lelong N, Colombier ML, Sixou M, Musset AM, Goffinet F, Kaminski M (2010) Maternal periodontitis and the causes of preterm birth: the case-control Epipap study. J Clin Periodontol 37(1):37-45

27. Radnai M, Gorzo I, Nagy E, Urban E, Eller J, Novak T, Pal A (2005) Caries and periodontal state of pregnant women. Part II periodontal state. Fogorv Sz 98(3):101-106

28. Yang JL, Wang MS, Cheng AC, Pan KC, Li CF, Deng SX (2008) A simple and rapid method for extracting bacterial DNA from intestinal microflora for ERIC-PCR detection. World J Gastroenterol 14(18): 2872-2876

29. Jentsch H, Cachovan G, Guentsch A, Eickholz P, Pfister W, Eick S (2012) Characterization of Aggregatibacter actinomycetemcomitans strains in periodontitis patients in Germany. Clin Oral Investig 16(6): 1589-1597

30. Canakci V, Canakci CF, Yildirim A, Ingec M, Eltas A, Erturk A (2007) Periodontal disease increases the risk of severe pre-eclampsia among pregnant women. J Clin Periodontol 34(8):639-645

31. Loe H, Silness J (1963) Periodontal disease in pregnancy. I. Prevalence and severity. Acta Odontol Scand 21:533-551

32. Kornman KS, Loesche WJ (1980) The subgingival microbial flora during pregnancy. J Periodontal Res 15(2):111-122

33. Figuero E, Carrillo-de-Albornoz A, Martin C, Tobias A, Herrera D (2013) Effect of pregnancy on gingival inflammation in systemically healthy women: a systematic review. J Clin Periodontol 40(5): $457-473$

34. Gursoy M, Pajukanta R, Sorsa T, Kononen E (2008) Clinical changes in periodontium during pregnancy and post-partum. J Clin Periodontol 35(7):576-583

35. Lieff S, Boggess KA, Murtha AP, Jared H, Madianos PN, Moss K, Beck J, Offenbacher S (2004) The oral conditions and pregnancy study: periodontal status of a cohort of pregnant women. J Periodontol 75(1):116-126
36. Adriaens LM, Alessandri R, Sporri S, Lang NP, Persson GR (2009) Does pregnancy have an impact on the subgingival microbiota. J Periodontol 80(1):72-81

37. Bieri RA, Adriaens L, Sporri S, Lang NP, Persson GR (2013) Gingival fluid cytokine expression and subgingival bacterial counts during pregnancy and postpartum: a case series. Clin Oral Investig 17(1):19-28

38. Lindhe J, Attsfrom R (1967) Gingival exudation during the menstrual cycle. J Periodontal Res 2(3):194-198

39. O'Neil TC (1979) Maternal T-lymphocyte response and gingivitis in pregnancy. J Periodontol 50(4):178-184

40. Carrillo-de-Albornoz A, Figuero E, Herrera D, Bascones-Martinez A (2010) Gingival changes during pregnancy: II. Influence of hormonal xvariations on the subgingival biofilm. J Clin Periodontol 37(3):230-240

41. Mariotti A (1994) Sex steroid hormones and cell dynamics in the periodontium. Crit Rev Oral Biol Med 5(1):27-53

42. Kornman KS, Loesche WJ (1982) Effects of estradiol and progesterone on Bacteroides melaninogenicus and Bacteroides gingivalis. Infect Immun 35(1):256-263

43. Tsai CC, Chen KS (1995) A study on sex hormones in gingival crevicular fluid and black pigmented bacteria in subgingival plaque of pregnant women. Gaoxiong Yi Xue Ke Xue Za Zhi 11(5):265-273

44. Yokoyama M, Hinode D, Yoshioka M, Fukui M, Tanabe S, Grenier D, Ito HO (2008) Relationship between Campylobacter rectus and periodontal status during pregnancy. Oral Microbiol Immunol 23(1):55-59

45. Aagaard K, Ma J, Antony KM, Ganu R, Petrosino J, Versalovic J (2014) The placenta harbors a unique microbiome. Sci Transl Med 6(237):237-265

46. Oettinger-Barak O, Barak S, Ohel G, Oettinger M, Kreutzer H, Peled M, Machtei EE (2005) Severe pregnancy complication (preeclampsia) is associated with greater periodontal destruction. $\mathrm{J}$ Periodontol 76(1):134-137

47. Chaparro A, Sanz A, Quintero A, Inostroza C, Ramirez V, Carrion F, Figueroa F, Serra R, Illanes SE (2013) Increased inflammatory biomarkers in early pregnancy is associated with the development of preeclampsia in patients with periodontitis: a case control study. J Periodontal Res 48(3):302-307

48. Sato TA, Keelan JA, Mitchell MD (2003) Critical paracrine interactions between TNF-alpha and IL-10 regulate lipopolysaccharidestimulated human choriodecidual cytokine and prostaglandin E2 production. J Immunol 170(1):158-166

49. Fortunato SJ, Menon R, Lombardi SJ (1998) The effect of transforming growth factor and interleukin-10 on interleukin-8 release by human amniochorion may regulate histologic chorioamnionitis. Am J Obstet Gynecol 179(3 Pt 1):794-799

50. Gotsch F, Romero R, Kusanovic JP, Erez O, Espinoza J, Kim CJ, Vaisbuch E, Than NG, Mazaki-Tovi S, Chaiworapongsa T, Mazor M, Yoon BH, Edwin S, Gomez R, Mittal P, Hassan SS, Sharma S (2008) The anti-inflammatory limb of the immune response in preterm labor, intra-amniotic infection/inflammation, and spontaneous parturition at term: a role for interleukin-10. J Matern Fetal Neonatal 21(8): $529-547$

51. Cobo T, Kacerovsky M, Holst RM, Hougaard DM, Skogstrand K, Wennerholm UB, Hagberg H, Jacobsson B (2012) Intra-amniotic inflammation predicts microbial invasion of the amniotic cavity but not spontaneous preterm delivery in preterm prelabor membrane rupture. Acta Obstet Gynecol Scand 91(8):930-935

52. Cobo T, Kacerovsky M, Palacio M, Hornychova H, Hougaard DM, Skogstrand K, Jacobsson B (2012) A prediction model of histological chorioamnionitis and funisitis in preterm prelabor rupture of membranes: analyses of multiple proteins in the amniotic fluid. J Matern Fetal Neonatal 25(10):1995-2001

53. Tsiartas P, Holst RM, Wennerholm UB, Hagberg H, Hougaard DM, Skogstrand K, Pearce BD, Thorsen P, Kacerovsky M, Jacobsson B (2012) Prediction of spontaneous preterm delivery in women with threatened preterm labour: a prospective cohort study of multiple proteins in maternal serum. BJOG 119(7):866-873 